\title{
Dual-Stage Feed Drive for Precision Positioning on Milling Machine
}

\author{
Hendra Prima Syahputra, Hyeon Mo Yang, Byeong Mook Chung, and Tae Jo Ko* \\ School of Mechanical Engineering, Yeungnam University, 214-1 Daedong, Gyoungsan, \\ 712-749, South Korea \\ tjko@yu.ac.kr
}

\begin{abstract}
A positioning system with long stroke and high precision by dualstage control was designed. The focus was on combining the long-stroke travel range of linear stage with high-precision characteristics of piezoelectric actuators. The control method used in the system was dynamic compensation type, where the error from linear stage becomes the input to the piezoelectric actuators. Positioning error of $30 \mu \mathrm{m}$ with single-stage control in a $1 \mathrm{~mm}$ stroke can be reduced to $2 \mu \mathrm{m}$ by using dual-stage control. This technique can be implemented for high-precision positioning in manufacturing and machining systems.
\end{abstract}

Keywords: Dual-stage, Long stroke, Dynamic compensation, Linear stage, Piezoelectric actuators.

\section{Introduction}

The need for high-precision parts has recently become an important concern in the manufacturing industry. Such a machine is required to provide versatility, speed and workspace as well as high-precision positioning [1]. This requirement impacts on machine tool design by improving machining performance, especially in terms of accuracy and precision. Generally, the positioning resolution of feed drive systems of such machine tools should be several times smaller than the required accuracy tolerance [2].

The most common and popular drive system used in a machine tool is the linear stage system. It has high load capacity and simple configuration for long stroke positioning application. Linear stage positioning accuracy depends on a number of factors, for instance their control electronics, motors, mechanical parts, and transmission configuration. For this reason, linear stages have several limitations such as: transmission errors, dead zone, backlash, elasticity, large inertia and wear [3]. This transmission drive is not a good option for short travel as it has high tendency for building high inertia, which leads to power loss in motor.

Positioning systems based on piezoelectric materials have received increased attention recently in many high-precision applications [1]. They have advantages that include: unlimited resolution, large force, fast expansion and no magnetic effects [4]. 
These characteristics make piezoelectric actuators a good choice for precision actuator. The disadvantages of piezoelectric actuators are their short travel range and nonlinearity.

The main goal of dual-stage actuation in this work was to obtain overall system that has the travel range of linear stage (coarse stage) and the accuracy of piezoelectric actuators (fine stage). The dual-stage control method proposed in this paper applies the concept of dynamic compensation, where the error from linear stage becomes the input of piezoelectric actuators in synchronous motion. This method is accepted for continuous path motion as well as point-to-point motion. To analyze the positioning performance, experiments were carried out for linear motion and circular motion to map the overall performance of the system.

\section{The Dual-Stage Mechanism}

\subsection{Design of Microstage for Fine Motion}

Although piezoelectric devices can provide large output forces, the typical $15 \mu \mathrm{m}$ displacement of stack-type actuator is not sufficient for most general engineering applications, for which much larger movement ranges $(0.05-0.5 \mathrm{~mm}$ or more) are typically required. In most cases, therefore, the use of a flexure-hinge mechanical displacement amplifier is the most appropriate approach to magnifying the output displacement of the piezoelectric actuators [5]. A microstage with flexure-hinge-type lever mechanisms was designed to increase the workspace of fine-stage mechanism. Simulation results for the micro-stage are shown in the Fig. 1.

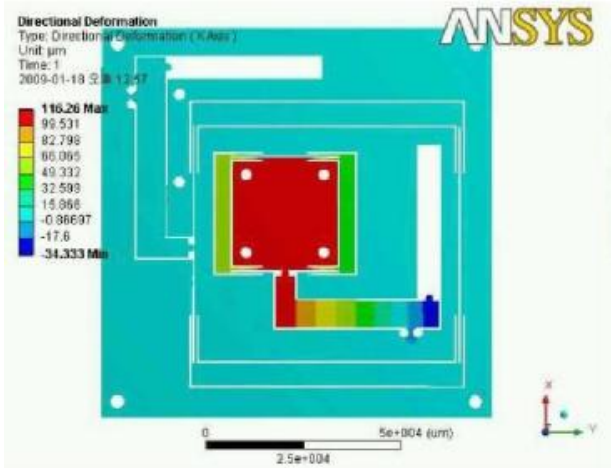

(a)

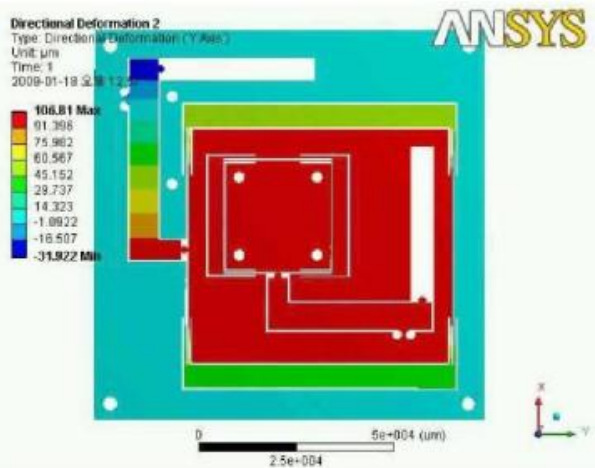

(b)

Fig. 1. Microstage simulation; (a) $x$-axis deformation; (b) $y$-axis deformation

Piezoelectric actuators used in the micro-stage were PI-830.20 made by Physik Instrumente. They had a travel range of $30 \mu \mathrm{m}$ and a $1000 \mathrm{~N}$ pushing force with a voltage range of 0 to $100 \mathrm{~V}$. With the help of the lever mechanism, the maximum travel ranges of the microstage become $116.26 \mu \mathrm{m}$ and $106.81 \mu \mathrm{m}$ for the $x$ - and $y$ axes, respectively. 


\subsection{The Developed System}

A standard 3-axis milling machine was modified by stacking the piezoelectric microstage on top of the $x-y$ (horizontal) working table, as shown in Fig. 2. The linear stages used 50000 pulse/rev stepping motors with $8 \mathrm{~mm}$ ball screw lead. The fine stage used $50 \mathrm{~nm}$ resolution linear encoders as a feedback for each $x$ - and $y$-axis in the microstage. A 2-D grid encoder with $156 \mathrm{~nm}$ resolution monitored the positioning error of the overall system.

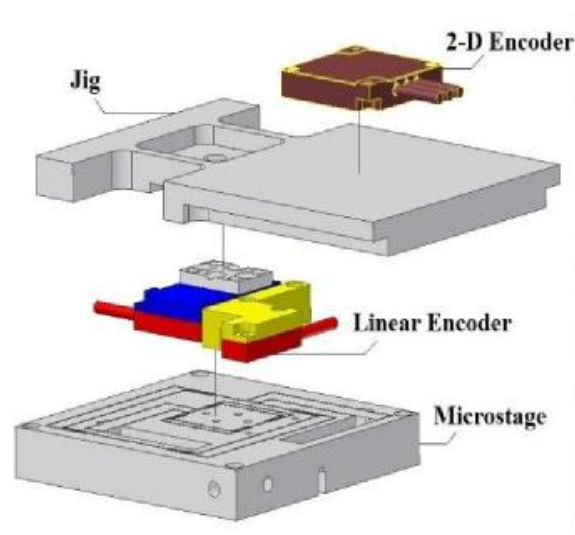

(a)

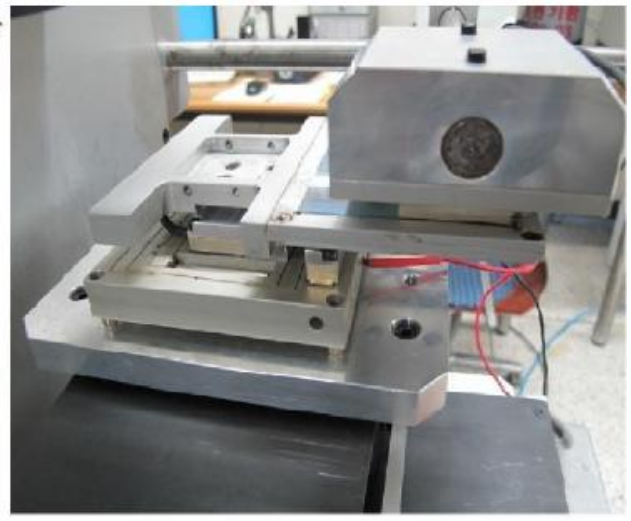

(b)

Fig. 2. Dual-stage milling machine configuration; (a) sensor arrangement; (b) assembly on the milling machine

The controller used for the dual-stage system was DeltaTau UMAC DSP. Position commands were generated on the controller with program code and sent to the stepping motor drivers and piezoelectric amplifier. The piezoelectric amplifier magnified command voltage by 20 times before passing it to the piezoelectric actuators.

\section{Control Strategy}

The control strategy used in the dual-stage feed drive system was dynamic compensation type, where an error from coarse stage becomes the input for the fine stage. The concept is to have compensation action in fine stage as the result of error generation in the coarse stage.

For the control law in the fine stage, a PI controller with notch filter was applied (Fig. 3). The parameter used in the controller was obtained by experiment and was tuned to compensate the nonlinearities characteristics of piezoelectric actuators. The performance was tested by step response analysis detected by linear encoders (Fig. 4). The initial position of $x$ - and $y$-axes in the microstage should be shifted approximately $50 \mu \mathrm{m}$ from the resting position in order to have compensation capability in both forward and backward direction. 
The fine stage works as an error compensator for the coarse stage which made the command for fine stage a deviation from commanded position and actual position of coarse-stage. The coarse stage was driven by open-loop, and the resulting control block diagram for the overall system is shown in Fig. 5.

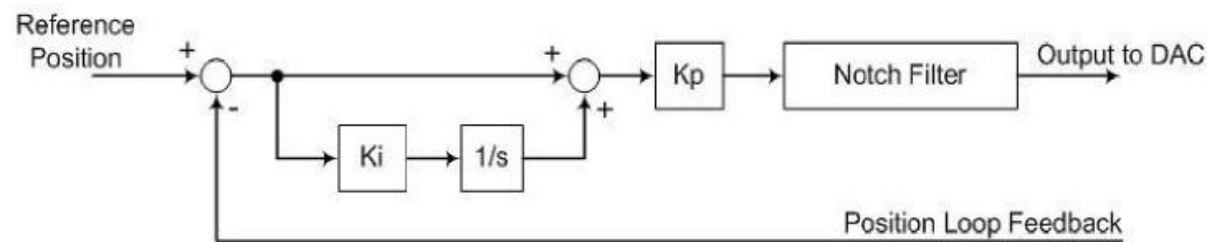

Fig. 3. Fine stage control block diagram

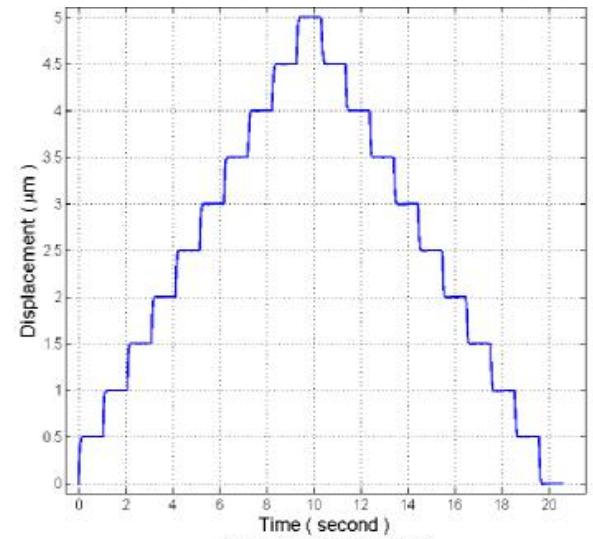

(a) X-axis step response

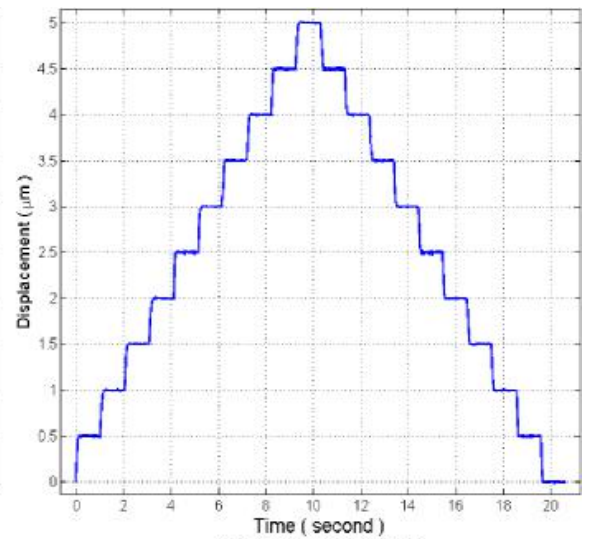

(b) Y-axis step response

Fig. 4. Microstage step response; (a) $x$ direction; (b) $y$ direction

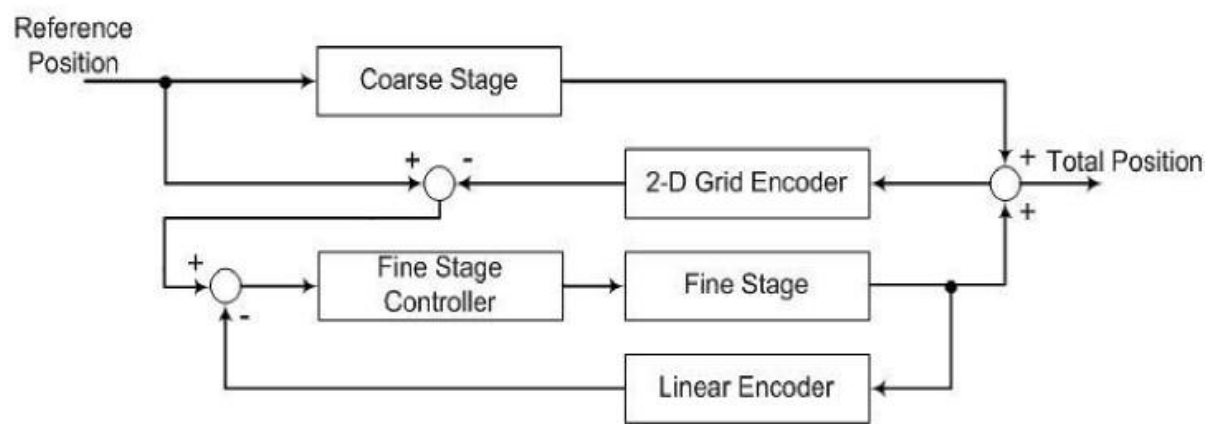

Fig. 5. Overall system control block diagram

The overall control block diagram shows the error compensation done for the total position uses the advantage of fine-stage fast response to compensate the error generation from the coarse-stage. 


\section{Experiment on the Milling Machine}

\subsection{Linear Trajectory Motion Test}

To perform the positioning experiment for linear trajectory, commanded trajectory for $1 \mathrm{~mm}$ back-and-forth motion was sent to linear stage with $4 \mu \mathrm{m} / \mathrm{s}$ constant speed. The linear trajectory motion experiment for $x$ - and $y$-axes was performed separately. For each $x$ - and $y$-axis, we tested the single-stage control first and then compared the result with the dual-stage control result. The results for the single-stage control are shown in the following in Fig. 6.
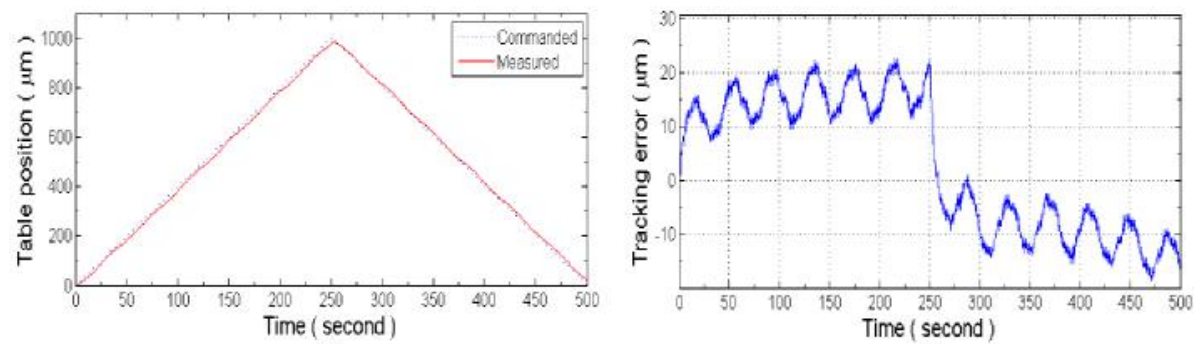

(a)

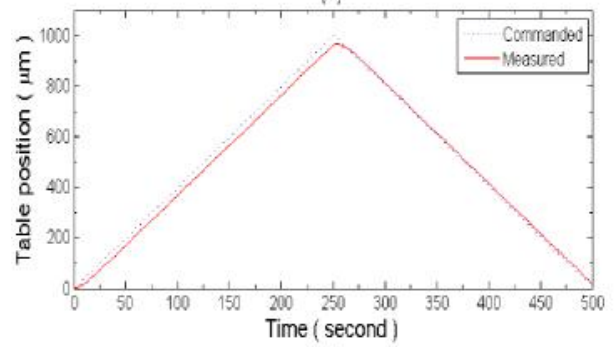

(c)

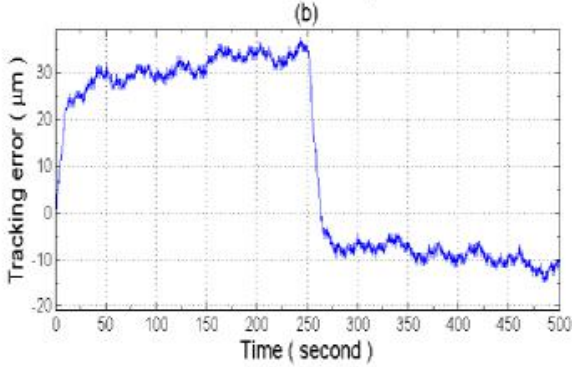

(d)

Fig. 6. Results of linear trajectory experiment by single-stage control; (a) $x$-axis commanded and measured position; (b) $x$-axis linear trajectory error map; (c) $y$-axis commanded and measured position; (d) $y$-axis linear trajectory error map

As shown in the graph, the magnitude of error along the $y$-axis was greater than along $x$-axis because in the milling machine the $x$-axis was stacked on top of the $y$ axis, which meant the $y$-axis experienced a larger load than the $x$-axis. The maximum positioning error in the $x$-axis was $22.51 \mu \mathrm{m}$, with $12.07 \mu \mathrm{m}$ overall error average. For the $y$-axis the maximum positioning error was $37.58 \mu \mathrm{m}$, with $19.60 \mu \mathrm{m}$ overall error average. However, these errors were still within the compensation range of the fine stage. The positive and negative signs of the error magnitude indicate the error direction. The most important concept is that the magnitude of error at any given time will affect the accuracy to a target point that needs to be achieved. This is an important consideration in the concept of point-to-point motion.

The analysis of the single-stage motion control confirmed that the errors generated from the linear stages were still in the compensation range of fine-stage. For this 
reason we performed a dual-stage experiment for linear trajectory. The results of linear trajectory motion with dual-stage control are shown in Fig. 7.

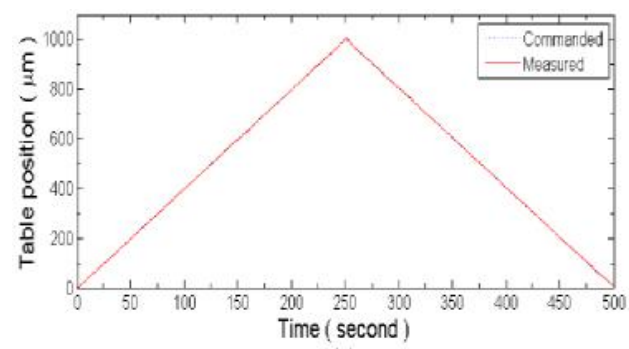

(a)

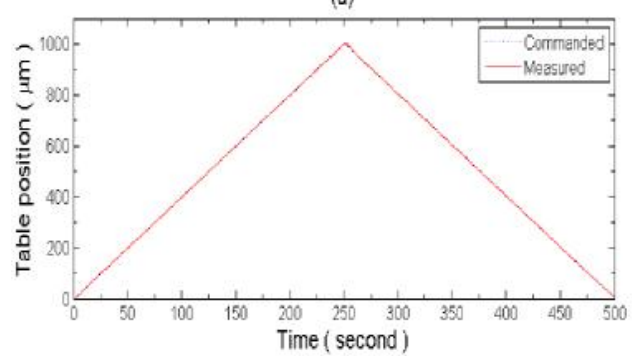

(c)

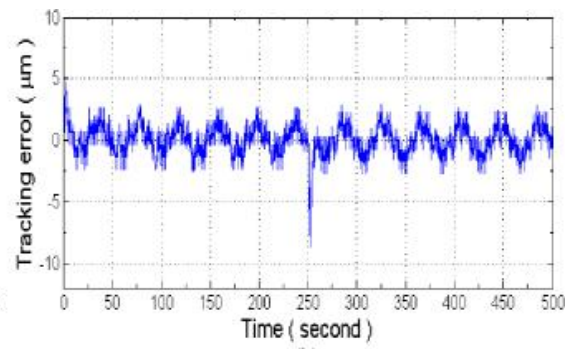

(b)

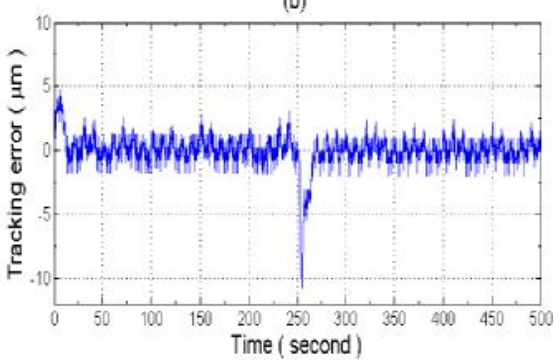

(d)

Fig. 7. Results of linear trajectory motion by dual-stage control; (a) $x$-axis commanded and measured position; (b) $x$-axis linear trajectory error map; (c) $y$-axis commanded and measured position; (d) $y$-axis linear trajectory error map

The graphs show error reduction in the $x$-axis for linear trajectory motion, although there were still some error peaks, in particular at the beginning of motion and when the motor reversed direction. Both conditions are times when the linear stage brings out the largest error because of the inertia in the system. The $x$-axis maximum positioning error with the dual-stage control was $8.67 \mu \mathrm{m}$, with $1.04 \mu \mathrm{m}$ overall error average. For the y-axis the maximum positioning error was $10.7 \mu \mathrm{m}$ with $0.93 \mu \mathrm{m}$ overall error average. This result indicated accuracy improvement by using the dualstage control.

The performance of the dual-stage control was defined largely by the relationship between the coarse and fine stage. Fine-stage responses also affect the overall performance of the system. The ability of the fine stage to respond error generation in the coarse stage will determine the performance of the dual-stage system.

\subsection{Circular Motion Test}

There are only two type of tool motion related to contouring in most milling machine applications. One is linear motion, which was discussed earlier, and the other is circular motion, which is discussed in this section.

Circular motion requires collaboration between two or even more linear axis. In our system we used $x$ - and $y$-axes to generate circular motion in $x-y$ plane. The interpolation command generated in the controller was to generate a circular motion 
with $1 \mathrm{~mm}$ radius at $4 \mu \mathrm{m} / \mathrm{s}$ constant speed. Experimental results for single-stage control and dual-stage control are shown in Figs 8 and 9.

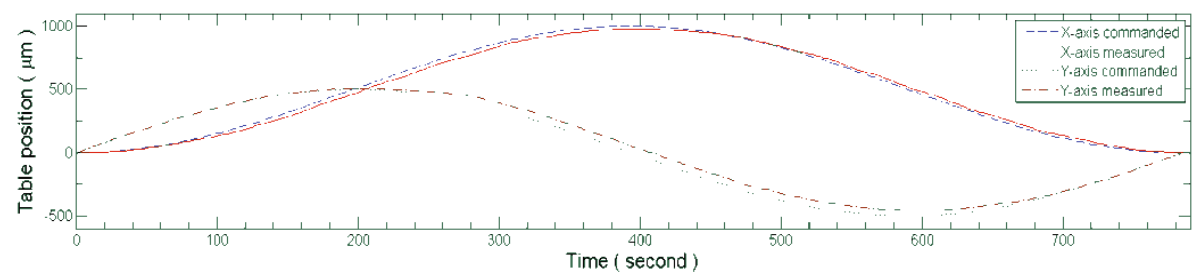

(a)

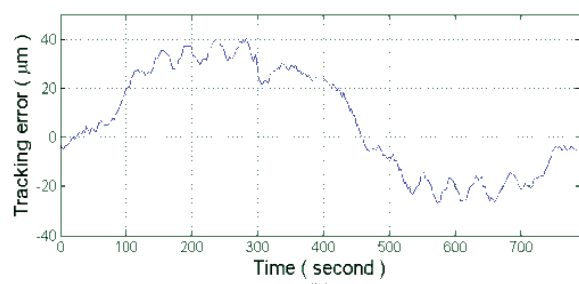

(b)

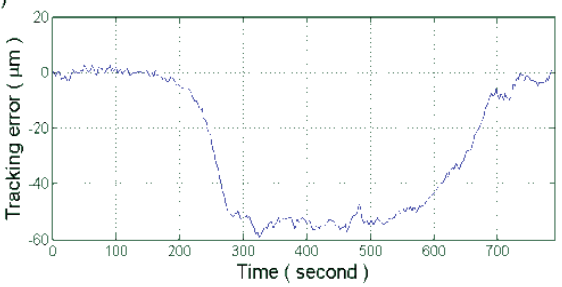

(c)

Fig. 8. Circular motion experiment results by single-stage control; (a) $x$ - and $y$-axes commanded and measured position; (b) $x$-axis circular trajectory error map; (c) $y$-axis circular trajectory error map

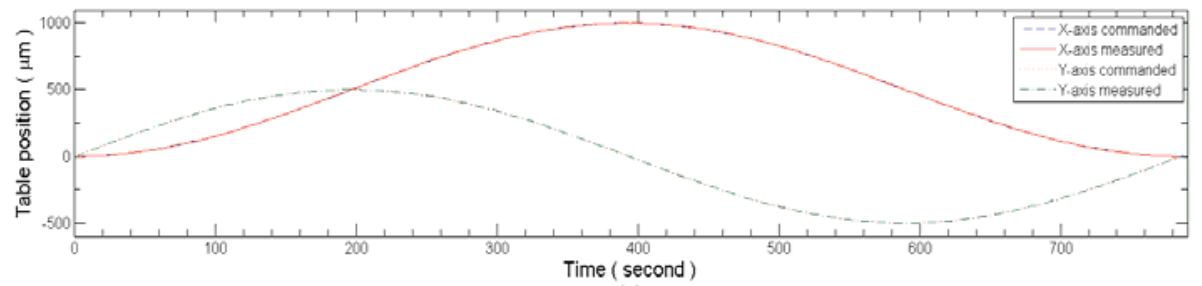

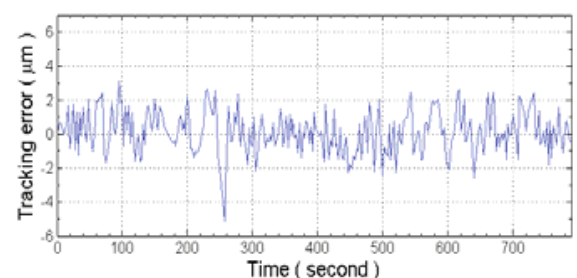

(b)

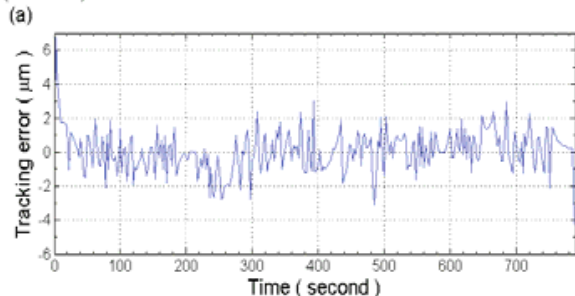

(c)

Fig. 9. Circular trajectory experiment results by dual-stage control; (a) $x$ - and $y$-axes commanded and measured position; (b) $x$-axis circular trajectory error map; (c) $y$-axis circular trajectory error map.

The maximum error on circular motion by single-stage control for $x$-axis was $40.73 \mu \mathrm{m}$ with $19.72 \mu \mathrm{m}$ overall error average. For the $y$-axis the maximum error was $59.06 \mu \mathrm{m}$ with $27.81 \mu \mathrm{m}$ overall error average. By using dual-stage control this error 
could be reduced with the maximum error in $x$-axis becoming $5.11 \mu \mathrm{m}$ with $0.995 \mu \mathrm{m}$ overall error average. For the $y$-axis the maximum error was reduced to $6.76 \mu \mathrm{m}$ with $0.93 \mu \mathrm{m}$ overall error average.

\section{Conclusion}

In this study, we developed a dual-stage feed drive mechanism and control strategy for a milling machine. The purpose was to have a combination of linear-stage longstroke capability and high-precision characteristics of piezoelectric actuators. The control strategy used for the dual-stage feed drive was dynamic compensation type where errors from linear stages become the input for piezoelectric actuators.

Experiments for linear and circular trajectory motion were carried out. We evaluated the positioning performance by comparing the performance of single-stage control with that of dual-stage control. In both cases there were significant improvements in machine accuracy by implementing a dual-stage feed drive mechanism. Therefore, this technique can be applied to develop high-precision positioning in the manufacturing and machining systems.

\section{References}

1. Elfizy, A.T., Bone, G.M., Elbestawi, M.A.: Design and Control of a Dual-stage Feed Drive. International Journal of Machine Tools \& Manufacture 45, 153-156 (2005)

2. Fujita, T., Matsubara, A., Kono, D., Yamaji, I.: Dynamic Characteristics and Dual Control of a Ball Screw Drive with Integrated Piezoelectric Actuator. Precision Engineering 34, 34-42 (2010)

3. Pritschow, G.: A Comparison of Linear and Conventional Electromechanical Drives. Annals of the CIRP 47(2), 541-548 (1998)

4. Ni, J., Zhu, Z.: Design of Linear Piezomotor with Ultra-high Stiffness and Nanoprecision. IEEE/ASME Transactions on Mechatronics 5(4), 441-443 (2000)

5. Xu, W., King, T.: Flexure Hinges for Piezoactuator Displacement Amplifiers: Flexibility, Accuracy and Stress Considerations. Precision Engineering 19, 4-10 (1996) 